OPEN ACCESS

Edited by:

Yusuf Tutar,

University of Health Sciences, Turkey

Reviewed by:

Andreina Bruno,

National Research Council (CNR), Italy

Tarek Mohamed Ali,

Beni-Suef University, Egypt

*Correspondence:

XiangYu Meng

mengxy@bjmu.edu.cn

YongFen Q

yongfenqi@163.com

Specialty section:

This article was submitted to

Clinical Diabetes,

a section of the journal

Frontiers in Endocrinology

Received: 15 July 2020 Accepted: 04 May 2021

Published: 19 May 2021

Citation:

Chai S, Chen Y, Xin S, Yuan N, Liu Y, Sun J, Meng $X$ and Qi Y (2021) Positive Association of Leptin and Artery

Calcification of Lower Extremity in

Patients With Type 2 Diabetes

Mellitus: A Pilot Study.

Front. Endocrinol. 12:583575.

doi: 10.3389/fendo.2021.583575

\section{Positive Association of Leptin and Artery Calcification of Lower Extremity in Patients With Type 2 Diabetes Mellitus: A Pilot Study}

\author{
SanBao Chai ${ }^{1}$, Yao Chen ${ }^{2,3,4}$, SiXu Xin ${ }^{1}$, Ning Yuan ${ }^{1}$, YuFang Liu ${ }^{1}$, JianBin Sun ${ }^{1}$, \\ XiangYu Meng ${ }^{5 *}$ and YongFen $Q i^{2,3,4 *}$ \\ ${ }^{1}$ Department of Endocrinology and Metabolism, Peking University International Hospital, Beijing, China, ${ }^{2}$ Laboratory of \\ Cardiovascular Bioactive Molecule, School of Basic Medical Sciences, Peking University, Beijing, China, ${ }^{3}$ Key Laboratory of \\ Molecular Cardiovascular Science, Ministry of Education, Peking University Health Science Center, Beijing, China, \\ ${ }^{4}$ Department of Pathogen Biology, School of Basic Medical Sciences, Peking University, Beijing, China, ${ }^{5}$ The Central \\ Laboratory, Peking University International Hospital, Beijing, China
}

Objective: We aimed to explore the role and possible mechanism of leptin in lowerextremity artery calcification in patients with type 2 diabetes mellitus (T2DM).

Methods: We recruited 59 male patients with T2DM and 39 non-diabetic male participants. All participants underwent computed tomography scan of lower-extremity arteries. The calcification scores (CSs) were analyzed by standardized software. Plasma leptin level was determined by radioimmunoassay kits. Human vascular smooth muscle cells (VSMCs) calcification model was established by beta-glycerophosphate and calcium chlorideinduction. Calcium deposition and mineralization were measured by the ocresolphthalein complexone method and Alizarin Red staining. The mRNA expression of bone morphogenic protein 2 (BMP2), runt-related transcription factor 2 (Runx2), osteocalcin (OCN) and osteopontin (OPN) was determined by quantitative RT-PCR. The protein levels of BMP2, Runx2, $\alpha$-smooth muscle actin ( $\alpha$-SMA) and (p)-Akt was determined by Western-blot analysis, and $\alpha$-SMA was also measured by immunofluorescence analysis.

Results: Compared with controls, patients with T2DM showed higher median calcification score in lower-extremity artery [286.50 (IQR 83.41, 1082.00) vs 68.66 (3.41, 141.30), $p<0.01]$. Plasma leptin level was higher in patients with calcification score $\geq 300$ than $\geq 100(252.67 \pm 98.57$ vs $189.38 \pm 44.19 \mathrm{pg} / \mathrm{ml}, p<0.05)$. Compared with calcification medium, intracellular calcium content was significantly increased in VSMCs treated by leptin (200, 400 and $800 \mathrm{ng} / \mathrm{ml}$ ) combined with calcification medium $[11.99 \pm 3.63,15.18 \pm 4.55$, and $24.14 \pm 5.85 \mathrm{mg} / \mathrm{ml}$, respectively, vs $7.27 \pm 1.54 \mathrm{mg} / \mathrm{ml}$, all $p<0.01]$. Compared with calcification medium, Alizarin Red staining showed calcium disposition was more obvious, and the mRNA level of BMP2, Runx2 and OCN was significantly increased, and immunofluorescence and Western blot analysis showed that the expression of $\alpha$-SMA was downregulated in VSMCs treated by leptin $(400 \mathrm{ng} / \mathrm{ml})$ 
combined with calcification medium, respectively. Compared with calcification medium, the protein level of BMP2 and Runx2 was upregulated in VSMCs treated by leptin (400 ng/ml) combined with calcification medium. Moreover, blocking PI3K/Akt signaling pathway can decrease the protein expression of BMP2 and Runx2 in VSMCs treated by leptin $(400 \mathrm{ng} / \mathrm{ml})$ combined with calcification medium.

Conclusions: Leptin promoted lower-extremity artery calcification of T2DM by upregulating the expression of BMP2 and Runx2, and regulating phenotypic switch of VSMCs via PI3K/AKt signaling pathway.

Keywords: leptin, type 2 diabetes mellitus, VSMC, artery calcification, phenotypic switch

\section{INTRODUCTION}

The global prevalence of diabetes in adults has increased in recent decades. The number of patients 20 to 79 years old with diabetes is predicted to increase to 642 million by 2040 (1). Arteriosclerotic calcification is increased in type 2 diabetes mellitus (T2DM), which impairing vascular compliance and function, thereby increasing the risk for heart attack, stroke, limb ischemia, and lower extremity amputation $(2,3)$. Arterial calcification has long been considered a passive process, but now it is known to be an active regulatory process of tissue biomineralization.

Recently, adipose tissue is recognized as a biologically active tissue. Adipose tissue is metabolically active and produces a multitude of adipokines, which may direct effects on the skeleton as well as on vascular structure and function (4). Previous reports indicated that abdominal obesity predicts coronary artery calcification presence and progression $(5,6)$. Clinical studies suggested that adipose tissue plays an important role in the formation of vascular calcification (VC) (7-9). Although the clinical effects of VC have been well documented, whether adipokines involved in the progression of VC is still unclear. Leptin, a $16-\mathrm{kDa}$ non-glycosylated protein, is encoded by the $o b$ gene and mainly secreted by adipocytes (10). The central function of leptin is to inhibit food intake and appetite, and the peripheral effects are mainly related to insulin resistance, inflammatory response, oxidative stress, atherosclerosis $(11,12)$. Plasma leptin level has a close relationship with the occurrence and development of obesity, metabolic syndrome and hypertension $(13,14)$. Previous studies implicated a link between high leptin concentrations and increased risk of cardiovascular as well as microvascular complications $(14,15)$. Vascular calcification can be divided into intimal calcification and media calcification in morphology, and media calcification is the most common type of vascular calcification in patients with T2DM. Oxidative stress, endothelial dysfunction, abnormal mineral metabolism and increased inflammatory factors are involved in the process of vascular calcification (16). Previous studies have found that adipokines play an important role in vascular calcification, and confirmed that leptin regulates the osteoblastic differentiation and calcification of vascular cells (17). Moreover, leptin is associated with insulin resistance and demonstrated as a significant independent predictor of coronary artery calcification (18).
The key link in the pathophysiological process of vascular calcification is the phenotypic transformation of smooth muscle cells into osteoblast like cells $(19,20)$. The differentiation of vascular smooth muscle cells (VSMCs) into osteoblast like cells mediates the process of bone matrix deposition in blood vessels.

Bone morphogenetic protein (BMP) is a member of the transforming growth factor superfamily and plays an important role in mammalian bone development. Among them, BMP2-Smad1/5/8 signaling pathway plays a vital role in ectopic ossification $(21,22)$. The Runt-related transcription factor (Runx2) is a key regulator of normal bone development, homeostasis and remodeling. During osteogenic differentiation, Runx2 activated by BMP2 regulate the expression of osteoblast markers such as osteocalcin (OCN) and osteopontin (OPN) (23, $24)$. The cytoskeletal $\alpha$-smooth muscle actin ( $\alpha$-SMA) is the actin isoform that predominates within VSMCs, which is one of the most well-established hallmarks and used to mark the phenotypic changes of myocytes $(25,26)$.

However, there is few information whether leptin is involved in the development of lower-extremity arterial calcification in T2DM. In the present study, we investigated changes of leptin level in T2DM with lower-extremity arterial calcification and its possible mechanisms involved in the development of T2DMrelated vascular calcification.

\section{MATERIALS AND METHODS}

\section{Materials}

Human VSMC CRL1999 were purchased from the American Type Culture Collection (ATCC, Manassas, VA, USA). Protease and phosphatase inhibitors were purchased from Roche Applied Science (Basel, Switzerland). Recombinant human leptin was obtained from R\&D Systems (398-LP-01, R\&D Systems, MN, USA). Anti-BMP2 antibody, anti- $\alpha$-SMA antibody, and antiglyceraldehyde-3-phosphate dehydrogenase (GAPDH) antibody were purchased from Abcam (Cambridge, United Kingdom). Anti-Akt antibody, Anti-p-Akt (Ser473) antibody, Anti-Runx2 antibody was purchased from Cell Signaling Technology Inc. (Beverly, MA, USA). LY294002 was obtained from Merck (Sigma-Aldrich, St. Louis, MO, USA). 4',6-diamidino-2phenylindole (DAPI), tetramethylrhodamine isothiocyanate (TRITC)-conjugated goat anti-rabbit, and horseradish 
peroxidase (HRP)-conjugated goat anti-rabbit secondary antibodies were purchased from Beijing Zhongshan Golden Bridge Biological Technology Company (Beijing, China).

\section{Study design}

All participants gave their informed consent for inclusion before they participated in the study. The study was conducted in accordance with the ethical guidelines of the 1975 Declaration of Helsinki, and the protocol was approved by the Ethics Committee of Peking University International Hospital (No. 2016-025). Participants had the right to refuse to participate in or withdraw from the study at any time. From January 2017 to December 2017, we recruited male participants with T2DM and non-diabetic male participants. The inclusion criteria of the diabetes group were males with $\mathrm{T} 2 \mathrm{DM} \geq 18$ years old receiving oral hypoglycemic agents with or without insulin. Exclusion criteria were previous bypass surgery or percutaneous angioplasty to lower-limb arteries, acute limb ischemia, moderate or severe renal disease, significant hepatic disease, cardiorespiratory disease, malignancy, connective tissue disease, smoking, drinking, uncontrolled hypertension, or acute illness.

\section{Calcification Scores of Lower-Extremity Arteries}

All participants underwent CT scan of lower extremity arteries with helical acquisition at $\mathrm{kVp}=120, \mathrm{mAs}=200$, and field of view 350 to $380 \mathrm{~mm}$. From the acquired raw data, the scan was reconstructed in 5-mm-thick slices. The average number of slices of lower-extremity arteries was approximately 250 . The scores for vascular calcification included external iliac, internal iliac, and superficial femoral arteries; deep femoral artery; popliteal arteries and peroneal arteries. Calcification scores (CSs) were analyzed by using standardized calcium scoring software (Intelligent portal space, Intellispace Portal, Philips Healthcare) by investigators who were blinded to clinical data. On crosssectional images of the lower extremities, the area of calcification in a cross-sectional area $>1 \mathrm{~mm}^{2}$ and density $>130$ hounsfield units was identified and scored. The CSs for each segment of interest was determined and expressed as an Agatston score (27).

\section{Sample Collection}

Blood samples were collected from all participants on an empty stomach for 8 hours. Ethylene diamine tetraacetic acid-plasma was isolated by centrifugation at 3,000 rpm for $15 \mathrm{~min}$. Aprotinin (500 units/ml; Sigma Co., St. Louis, MO, USA) was added into plasma for leptin analysis and samples were stored at $-80^{\circ} \mathrm{C}$.

\section{Plasma Radioimmunoassay}

Plasma leptin level was determined by using commercially available radioimmunoassay kits (DIAsource, Belgium). According to the manufacturer's protocol, the radioactive precipitation was counted by using a $\gamma$-counter. The intra- and inter-assay coefficients of variation were $<10 \%$ and $15 \%$.

\section{Measurement of Other Parameters}

Blood pressure was measured in the supine position for all participants. Levels of fasting blood glucose (FBG), high- density lipoprotein cholesterol (HDL-C), low-density lipoprotein cholesterol (LDL-C), triglyceride (TG), total cholesterol (TC), and glycosylated hemoglobin $\mathrm{Al}_{\mathrm{C}}\left(\mathrm{HbAl}_{\mathrm{C}}\right)$ were measured by routine biochemistry analysis.

\section{Cell Culture of Human VSMCs}

Human VSMC CRL1999 were purchased from the American Type Culture Collection (ATCC, Manassas, VA, USA) and cultured in Dulbecco's modified Eagle's medium supplemented with $15 \%$ fetal bovine serum (Gibco, Life Technologies), $100 \mathrm{U} /$ $\mathrm{ml}$ penicillin and $100 \mu \mathrm{g} / \mathrm{ml}$ streptomycin in a humidified atmosphere containing $5 \% \mathrm{CO}_{2}$. VSMCs at passages 7-10 were used for the experiments.

\section{In Vitro Calcification and Quantification of VSMCs}

VSMCs were cultured with calcification medium containing calcium chloride $(5 \mathrm{mmol} / \mathrm{l}), \beta$-glycerophosphate $(10 \mathrm{mmol} / \mathrm{l})$ and leptin $(200,400$ and $800 \mathrm{ng} / \mathrm{ml}$ ) or calcification medium alone. The calcification medium was replaced every 3 days for a total of 7 days. VSMCs were decalcified for $6 \mathrm{hr}$ at $4^{\circ} \mathrm{C}$ in $0.6 \mathrm{~mol} /$ $1 \mathrm{HCl}$ and calcium levels were determined by colorimetry with the o-cresolphthalein complexone method (Calcium Kit, Biosino Bio-Technology and Science, Beijing). $\mathrm{Ca}^{2+}$ concentration (mg/ $\mathrm{ml}$ ) in samples was normalized over the sample protein concentration $(\mathrm{mg} / \mathrm{ml})$ as determined by the Lowry assay.

\section{Alizarin-Red Staining}

Calcification of human VSMCs was induced as previously described (28) with minor modification. After removing the medium, VSMCs grown in 12-well plates were washed with phosphate-buffered saline (PBS), fixed with $4 \%$ formaldehyde, and stained with alizarin red for $5 \mathrm{~min}$, then washed again with distilled water and observed by microscopy.

\section{Immunofluorescence Analysis}

Human VSMCs were fixed with $4 \%$ formaldehyde for $10 \mathrm{~min}$, washed 3 times in PBS, permeabilized with $0.5 \%$ Triton X-100 for $15 \mathrm{~min}$ at room temperature, then preincubated with normal goat serum for $30 \mathrm{~min}$ and incubated with anti- $\alpha$-SMA antibody (1:200 in PBS) overnight at $4^{\circ} \mathrm{C}$. Then, cells were washed 3 times and incubated with TRITC conjugated goat anti-rabbit (594 nm, 1:200) antibodies for $1 \mathrm{~h}$. Nuclei were stained using DAPI (1:1000 diluted in PBS) for $5 \mathrm{~min}$. Cells on coverslips were then mounted on slides and examined with fluorescence microscope (Nikon).

\section{Quantitative RT-PCR (qRT-PCR)}

qRT-PCR was used to determine the effect of leptin on the expression of bone-related gene markers in VSMCs. Total RNA was isolated from cultured VSMCs by using TRIzol Reagent (Invitrogen, Carlsbad, CA, USA) according to the manufacturer's instructions. Reverse transcription involved use of an RT-PCR kit (Takara Biotech). Levels of mRNA were quantified by using an ABI 7500HT Fast Real-Time PCR System (Applied Biosystems, Grand Island, NY, USA). GAPDH was an endogenous control. The $2^{-\Delta \Delta C t}$ method was used to calculate relative expression level. See Table 1 for specific primers. 
TABLE 1 | Primers and protocols for qRT-PCR.

\begin{tabular}{ll}
\hline Genes & \multicolumn{1}{c}{ Primer sequence } \\
\hline Human BMP2 & Fwd: TCAAGCCAAACACAAACAGC \\
Human Runx2 & Rev: GGAGCCACAATCCAGTCATT \\
Human OCN & Rev: ATGAAATGCTTGGGAACTGC \\
Human OPN & Fwd: CTCACACTCCTCGCCCTATT \\
Human GAPDH & Rev: CGCCTGGGTCTCTTCACTAC \\
& Fwd: GCCGTGGGAAGGACAGTTAT \\
& Rev: GCTCATTGCTCTCATCATTGG \\
& Fwd: CAGGAGGCATTGCTGATGAT \\
& Rev: GAAGGCTGGGGCTCATTT
\end{tabular}

BMP2, bone morphogenic protein 2; Runx2, runt-related transcription factor 2; OCN osteocalcin; OPN, osteopontin; GAPDH, glyceraldehyde-3-phosphate dehydrogenase.

\section{Western Blot Analysis}

Protein extraction from cultured human VSMCs was prepared in radioimmunoprecipitation assay buffer supplemented with protease inhibitor and phosphatase inhibitor. Total protein was subjected to electrophoresis on sodium dodecyl sulfatepolyacrylamide gel electrophoresis gels and electrically transferred to nitrocellulose membranes. Then, membranes were blocked in 5\% nonfat milk for 1 hour and incubated overnight at $4^{\circ} \mathrm{C}$ with the following primary antibodies: antiBMP2 (1:200 dilution), anti-Runx2 (1:200 dilution), anti-Akt (1:1000 dilution), anti-p-Akt (1:1000 dilution), anti- $\alpha$-SMA (1:1000 dilution), and anti-GAPDH (1:2000 dilution). The membranes were then incubated with the HRP-conjugated goat antirabbit immunoglobulin G secondary antibody for 1 hour, followed by detection with ECL (Millipore).

\section{Statistical Analysis}

SPSS 23 (SPSS, Chicago, IL, USA) was used for statistical analysis. Data are tested for normality by Shapiro-Wilk test and expressed as mean $\pm \mathrm{SD}$ or median (interquartile range $[\mathrm{IQR}]$ ). Statistical analysis included independent $t$-test, paired $t$-test for normal distribution and Mann-Whitney $U$ test for non-normal distribution. $p<0.05$ was considered statistically significant.

\section{RESULTS}

\section{Increased Lower-Extremity Artery Calcification Score and Leptin Level in Patients With T2DM}

We included 59 males patients with T2DM and 39 non-diabetic male participants. As compared with controls, patients with T2DM showed higher median calcification score in lowerextremity artery [286.50 (IQR 83.41, 1082.00) vs 68.66 (3.41, $141.30), p<0.01]$, and median plasma leptin level [183.40 (130.00, 248.80) vs $139.00(82.36,218.30) \mathrm{pg} / \mathrm{ml}, p<0.05]$ (Table 2).

\section{Plasma Leptin Level Associated With Severity of Lower-Extremity Arterial Calcification in T2DM}

Participants with T2DM were divided into two groups by lowerextremity arterial calcification scores: $\geq 100$ and $\geq 300$. Mean plasma leptin level was higher with calcification score $\geq 300$ than $\geq 100(252.67 \pm 98.57$ vs $189.38 \pm 44.19 \mathrm{pg} / \mathrm{ml}, p<0.05)$ (Figure 1A). The correlation coefficient $\mathrm{R}^{2}$ was 0.2184 between plasma leptin and vascular calcification score (Figure 1B). These results indicated that plasma leptin level associated with severity of lower-extremity arterial calcification in T2DM.

\section{Leptin Promotes VSMCs Calcification In Vitro}

To investigate whether leptin could promote vascular calcification, human VSMCs were treated with various concentrations of leptin combined with calcification medium for 7 days. Calcium content was significantly increased with calcification medium combined with leptin 200, 400 and $800 \mathrm{ng} /$ $\mathrm{ml}$ versus calcification medium alone $[11.99 \pm 3.63,15.18 \pm 4.55$, and $24.14 \pm 5.85 \mathrm{mg} / \mathrm{ml}$, respectively, vs $7.27 \pm 1.54 \mathrm{mg} / \mathrm{ml}$, all $p<0.01]$ (Figure 2A). After treated with leptin $(400 \mathrm{ng} / \mathrm{ml})$ and calcification medium for 7 days, Alizarin Red staining showed calcium disposition was more obvious than that in calcification medium alone (Figure 2B).

\section{Leptin Induced Phenotypic Switch of VSMCs In Vitro}

To test whether leptin promoted vascular calcification involving in phenotypic switch of VSMCs, marker of VSMCs contractile phenotype was measured. Compared with calcification medium alone, immunofluorescence and Western blot analysis showed that the expression of $\alpha$-SMA decreased in human VSMCs incubated with leptin and calcification medium. The results were shown in Figures 3A, B. The result indicated that leptin promoted phenotypic switch of VSMCs by decreasing the expression of $\alpha$-SMA.

\section{Leptin Regulated the Expression of Bone- Related Gene or Protein Markers via PI3K/ Akt Signaling Pathway in VSMCs In Vitro}

To explore the possible mechanism of leptin promoting vascular calcification, the markers of bone-related gene or protein were measured. The mRNA level of BMP2, Runx 2 and OCN was significantly increased by $68 \%(p<0.01), 27 \%(p<0.05)$ and $23 \%$ $(p<0.05)$ in VSMCs incubated with leptin and calcification medium versus calcification medium alone. And the mRNA level of OPN showed no significance in leptin with calcification medium versus calcification medium alone (Figure 4A). As compared with calcification medium alone, treatment with leptin and calcification medium increased the protein level of BMP2 and Runx2 (Figure 4B). Moreover, the phosphorylation of Akt was upregulated in VSMCs treated with leptin and calcification medium than calcification medium alone (Figure 4C).

To examine whether PI3K/Akt signaling pathway involved in the regulation of BMP2 and Runx2, LY294002, the specific inhibitor of PI3K/Akt, was used in further study. As shown in Figure 4D, LY294002 decreased the protein expression of Runx2 and BMP2 induced by leptin. These results demonstrated that leptin aggravated vascular calcification by increasing expression of bone related gene or protein via PI3K/Akt signaling pathway. 
TABLE 2 | Baseline characteristics of patients with type 2 diabetes mellitus and control group.

\begin{tabular}{|c|c|c|c|}
\hline parameter & T2DM group & control group & $p$ value \\
\hline $\mathrm{N}$ & 59 & 39 & - \\
\hline Age $^{\star}$ & $55.81 \pm 14.74$ & $53.74 \pm 16.05$ & 0.51 \\
\hline Duration (year) ${ }^{\#}$ & $7.00(2.00,13.00)$ & - & - \\
\hline BMl $\left(\mathrm{kg} / \mathrm{m}^{2}\right)^{\#}$ & $26.10(23.90,28.60)$ & $25.00(24.10,26.10)$ & 0.11 \\
\hline $\operatorname{SBP}(\mathrm{mmHg})^{\#}$ & $135.00(123.00,145.00)$ & $131.00(120.00,141.00)$ & 0.50 \\
\hline $\mathrm{DBP}(\mathrm{mmHg})^{\#}$ & $80.00(75.00,89.00)$ & $80.00(70.00,89.00)$ & 0.38 \\
\hline $\mathrm{FBG}(\mathrm{mmol} / \mathrm{L})^{\#}$ & $7.87(6.39,9.56)$ & $5.55(5.02,6.24)$ & $<0.01$ \\
\hline $\mathrm{HbAlc}(\%)^{\#}$ & $7.60(6.80,9.40)$ & $5.60(5.20,5.80)$ & $<0.01$ \\
\hline $\operatorname{ALT}(\mathrm{U} / \mathrm{L})^{\#}$ & $22.00(16.00,30.00)$ & $25.00(16.00,33.00)$ & 0.81 \\
\hline AST $(U / L)^{\#}$ & $20.00(16.00,28.00)$ & $23.00(20.00,30.00)$ & 0.06 \\
\hline Cholesterol (mmol/L) & $4.18(3.35,4.77)$ & 3.96(3.52, 4.79) & 0.98 \\
\hline Triglyceride $(\mathrm{mmol} / \mathrm{L})^{\#}$ & $1.28(0.90,2.13)$ & $1.36(1.10,1.90)$ & 0.63 \\
\hline HDL-C (mmol/L) $)^{\#}$ & $0.89(0.78,1.10)$ & $0.96(0.83,1.04)$ & 0.26 \\
\hline LDL-C (mmol/L) & $2.54(1.94,3.00)$ & $2.42(2.01,3.08)$ & 0.97 \\
\hline Leptin $(p g / m l)^{\#}$ & $183.40(130.00,248.80)$ & 139.00(82.36, 218.30) & $<0.05$ \\
\hline calcification scores $^{\#}$ & 286.50(83.41, 1082.00) & 68.66(3.41, 141.30) & $<0.01$ \\
\hline
\end{tabular}

T2DM, type 2 diabetes mellitus; BMI, body mass index; SBP, systolic blood pressure; DBP, diastolic blood pressure; FBG, fasting blood glucose; HbA1C, glycated hemoglobin A1 ; ALT, alanine aminotransferase; AST, aspartate aminotransferase; HDL-C, high-density lipoprotein cholesterol; LDL-C, low-density lipoprotein cholesterol.

*data expressed as mean $\pm S D$ and tested by independent $t$-test for the normal distribution.

\#data expressed as median (p25, p75) and tested by Mann-Whitney $U$ test for the non-normal distribution.

\section{DISCUSSION}

Vascular calcification is a characteristic change of diabetic vascular disease, including intimal and media calcification. Previous studies showed VC is the main independent risk factor for morbidity and mortality of cardiovascular diseases with diabetes $(29,30)$. Hyperglycemia and increased glycation end products, hyperinsulinemia, oxidative stress, lipid metabolism disturbance, inflammation, apoptosis and abnormal expression of bone regulatory protein are the main factors of vascular calcification in T2DM, which are closely related to the occurrence and development of vascular calcification $(31,32)$. In a population-based multi-ethnic cohort (33), Katz et al. demonstrated that both metabolic syndrome and diabetes are independently associated with increased prevalence and severity of calcified atherosclerotic plaque in the thoracic aorta. Consistent with our findings,
Chen et al. (34) demonstrated that the calcification of VSMCs is significantly enhanced when co-cultured with adipocytes compared to VSMCs alone. A recent study of 548 communitydwelling men reported high leptin levels associated with greater severity and rapid progression of abdominal aortic calcification, independent of smoking or LDL-C or TG levels (35). Moreover, a high baseline level of leptin was found associated with increasing coronary artery calcium severity (36). In according with our results, Tanna et al. (37) indicated, in a cohort of community dwelling post-menopausal women, adiponectin, leptin and vaspin regulate pathways linked with atherosclerosis, VC and stiffness. Wu et al. (38) found that the serum leptin levels were negatively associated with spine, hip and femur bone mineral density (BMD). In a meta-analysis, leptin was positively associated with BMD in post-menopausal women, although the associations were attenuated after adjusting for body mass index (39). It is expected to provide new ideas for the prevention
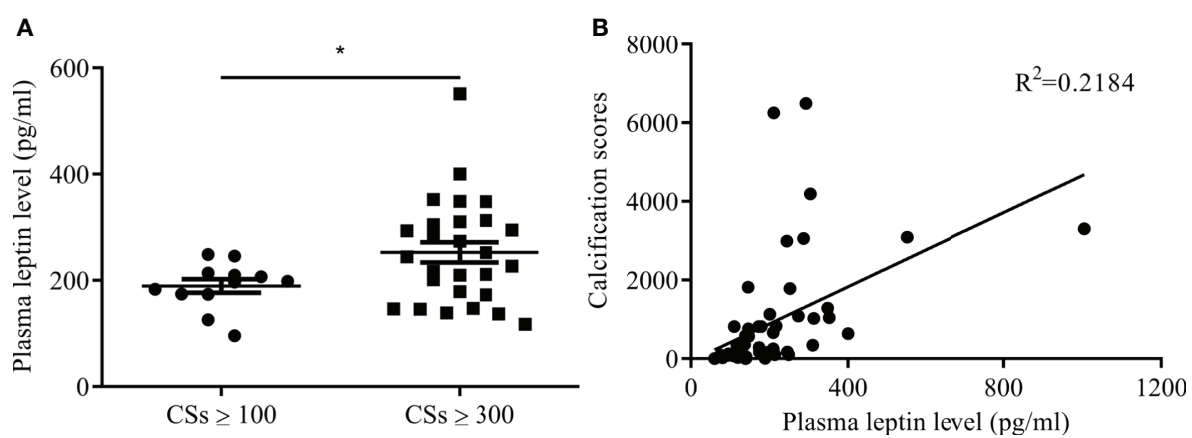

FIGURE 1 | Plasma leptin levels in patients with T2DM and arterial calcification of lower extremity. (A) plasma leptin levels between calcification score $\geq 100$ and $\geq 300$. Data expressed as median (p25, p75), $n=12$ CSs $\geq 100, n=29$ CSs $\geq 300$, ${ }^{*} p<0.05$ vs CSs $\geq 100$ (B) the correlation between plasma leptin levels and calcification scores. CSs, calcification scores; T2DM, type 2 diabetes mellitus. 


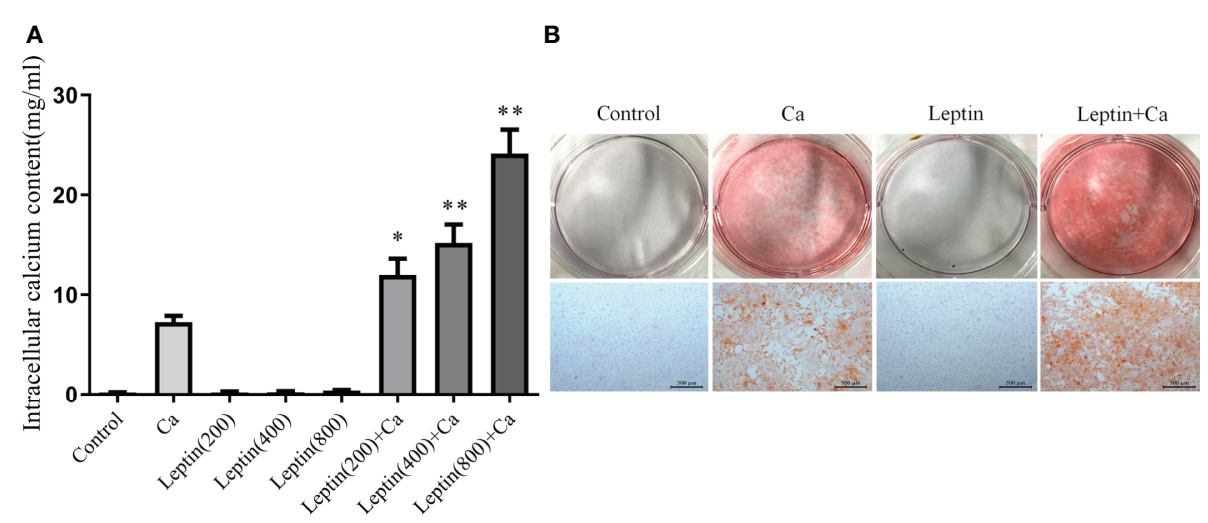

FIGURE 2 | Leptin enhances calcification of VSMCs. (A) intracellular calcium content. Data are mean \pm SD. $n=6$ independent experiments. ${ }^{*} p<0.05$, ${ }^{\star \star} p<0.01$ vs Ca. (B) alizarin red staining (Scale bar=500 $\mu \mathrm{m})$. VSMCs, vascular smooth muscle cells; Ca, calcification medium; Leptin (200), 200 ng/ml; Leptin (400), 400 ng/ml; Leptin (800), $800 \mathrm{ng} / \mathrm{ml}$.

of cardiovascular complications in T2DM by exploring the pathogenesis and relationship between VC and diabetes. In the present study, we found that patients with T2DM showed higher plasma leptin level and median calcification score in lowerextremity artery than control group. Moreover, the results of correlation analysis showed that plasma leptin level was positively correlated with the severity of calcification in T2DM $\left(R^{2}=0.2184\right)$. Our results suggested that plasma leptin could reflect the severity of vascular calcification of lower extremity. The VC process, analogous to that of bone morphogenesis, can generally be described as a form of progressive arterial stiffness that results in reduced vessel compliance and function, ultimately increasing the risk of limb ischemia and lowerextremity amputation $[(2,3,40,41)$. Historically, advancing VC was considered a passive deposition of calcium along the vessel wall. However, VC formation is now considered a complex, actively regulated process, similar to that required for skeletal bone formation.

Osteoblastic differentiation of VSMCs plays an important role in arterial calcification (42). With phenotype switching, VSMCs alternate from contractile (differentiated) phenotype to synthetic (dedifferentiated) phenotype, as well as reduced expression of
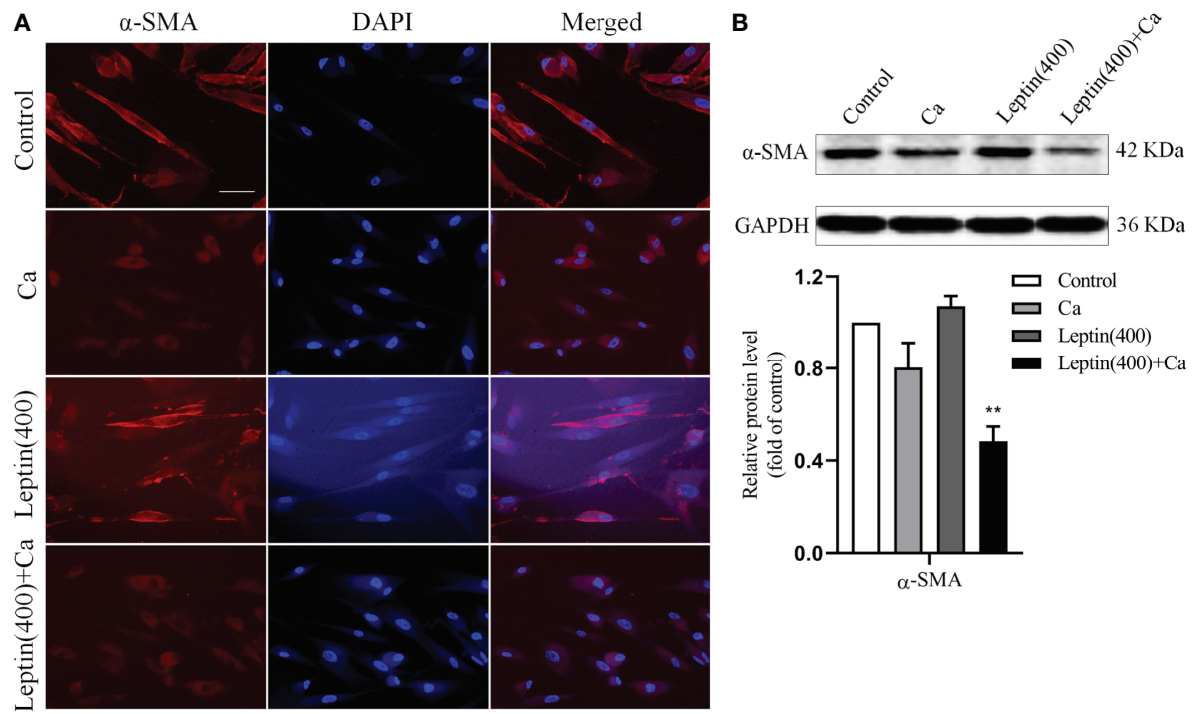

FIGURE 3 | Leptin induced phenotypic switch of VSMCs in vitro. (A) representative immunofluorescence staining with antibodies for $\alpha$-SMA in VSMCs (magnification: $\times 200$ ) (Scale bar $=25 \mu \mathrm{m})$. (B) Western blot assay for expression of $\alpha-S M A$ in VSMCs. Data are shown as mean \pm SD. $n=3$ independent experiments. ${ }^{\star *} p<0.01$ vs Ca. VSMCs, vascular smooth muscle cells; Ca, calcification medium; Leptin (400), 400 ng/ml; $\alpha$-SMA, $\alpha$-smooth muscle actin; DAPI, 4',6-diamidino-2phenylindole; GAPDH, glyceraldehyde-3-phosphate dehydrogenase. 

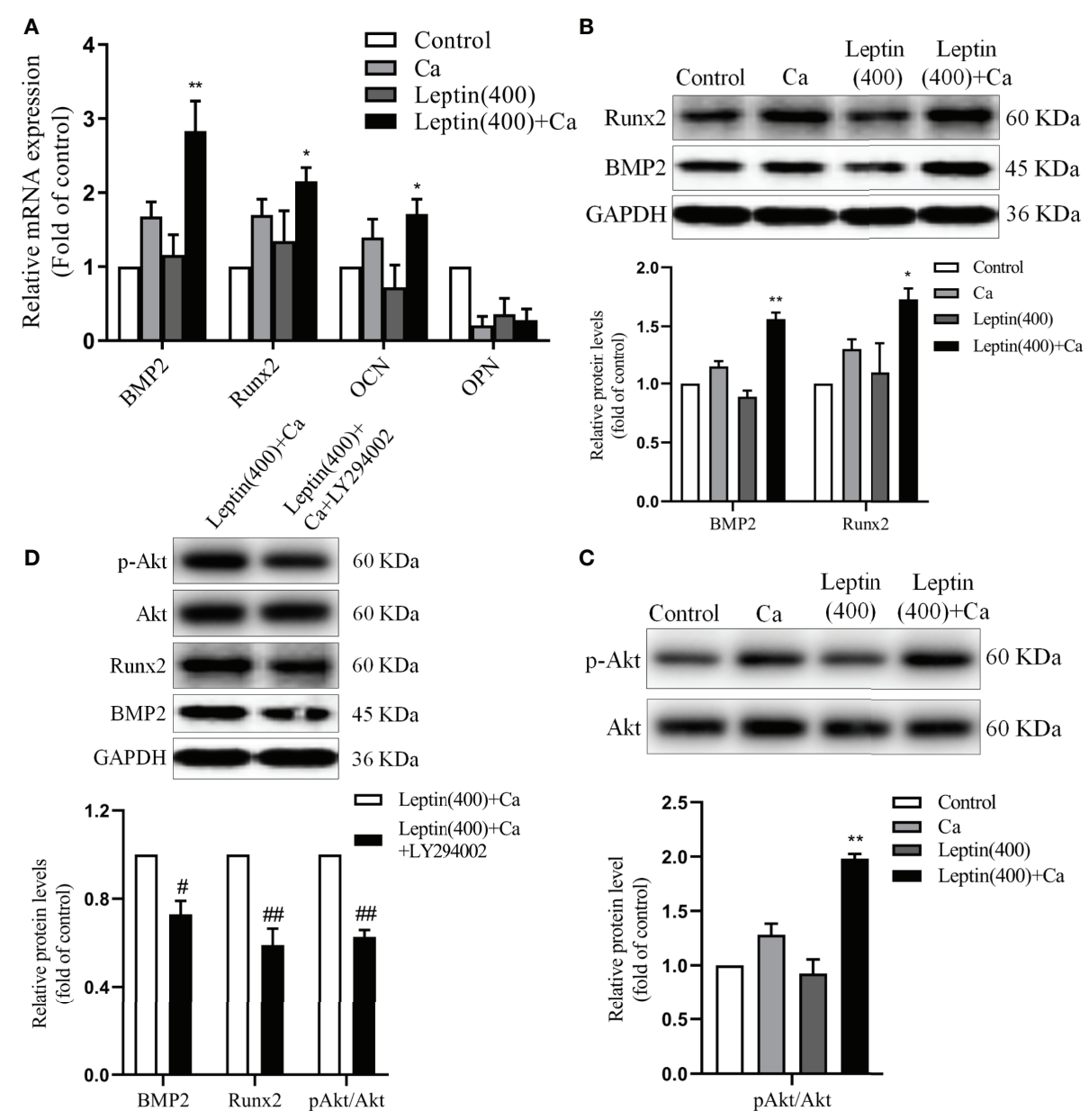

FIGURE 4 | Leptin regulates the expression of bone-related gene markers in VSMCs. (A) quantitative RT-PCR assay for expression of BMP2, Runx2, OCN and OPN in VSMCs. Data are mean \pm SD. $n=6$ independent experiments. ${ }^{*} p<0.05,{ }^{* *} p<0.01$ vs Ca. (B) Western blot assay for expression of BMP2 and Runx2 in VSMCs. Data are mean \pm SD. $n=3$ independent experiments. ${ }^{\star} p<0.05,{ }^{\star \star} p<0.01$ vs Ca. (C) Western blot assay for Akt and $p-A k t$ in VSMCs. Data are mean \pm SD. $\mathrm{n}=3$ independent experiments. ${ }^{* *} \mathrm{p}<0.01 \mathrm{vs}$ Ca. (D) Western blot assay for expression of Akt, $\mathrm{p}-\mathrm{Akt}$, BMP2 and Runx2 in VSMCs. Data are mean \pm SD. $\mathrm{n}=3$ independent experiments. ${ }^{\# p<0.05, ~}{ }^{\# \#} p<0.01$ vs Leptin(400)+Ca. VSMCs, vascular smooth muscle cells; Ca, calcification medium; Leptin (400), 400 ng/ml; BMP2, bone morphogenic protein 2; Runx2, runt-related transcription factor 2; OCN, osteocalcin; OPN, osteopontin; Akt, protein kinase B; GAPDH, glyceraldehyde-3phosphate dehydrogenase.

differentiation marker $\alpha$-SMA (43). In this study, we detected the expression of $\alpha$-SMA in calcified VSMCs model using immunofluorescence. The results showed that the fluorescence intensity of $\alpha$-SMA in leptin with calcification medium was significantly lower than calcification medium alone, Furthermore, compared with calcification medium alone, the protein expression of $\alpha$-SMA in leptin with calcification medium was decreased. Our results suggested that leptin aggravates the phenotype transition of VSMCs induced by calcification medium, which evidently contributed to the decreased expression of $\alpha$-SMA.

BMPs are a superfamily of transforming growth factor beta and secretory growth factor, which are reported to have osteogenic actions and play important roles in bone formation. Study reported that vascular calcification is associated with deposition of BMPs in patients with end-stage renal disease, which implied an active cell-mediated process and could be able to intervention (44). The combined use of leptin/leptin receptor and mechanical stress promotes osteogenic differentiation of the posterior longitudinal ligament, accompanied by upregulation of the osteogenic markers osteocalcin and Runx2 (45). Runx2, a key regulator of osteoblast differentiation and bone development, can induce transdifferentiation of VSMCs to an osteochondrocytic phenotype (46). But the exact mechanism of VC in T2DM is still unclear. In this study, we firstly used different concentrations of leptin on VSMCs calcification model. The results showed that leptin could further aggravate calcium deposition of VSMCs. Moreover, leptin with calcification medium could effectively increase the mRNA level of BMP2, Runx2 and OCN versus calcification medium alone in VSMCs. Western blot analysis 
indicated the protein expression of BMP2 and Runx2 was upregulated by leptin with calcification medium versus calcification medium alone in VSMCs. Consistent with our results, previous studies demonstrated that BMP2 and Runx2 play a crucial role in vascular calcification (46-48). PI3K/Akt signaling pathway was involved in a variety of biological process. In recent years, evidences showed that osteogenesis was regulated by PI3K/Akt signaling pathway (49-51). Our study indicated that inhibition of PI3K/Akt signaling pathway can reverse the expression of osteogenic makers induced by leptin, suggesting that leptin regulates the osteogenic transformation of VSMCs through PI3K/Akt signaling pathway.

The present study demonstrates that VSMCs incubated with leptin and a high calcium environment undergo phenotypic switch that led to some limited osteoblast-like properties. However, this comparison was for in vitro studies with calcification induced by increasing both leptin and extracellular calcium level. Ultimately, understanding the differences between VSMCs and osteoblasts may help in developing therapeutic strategies that prevent pathological vascular calcification in T2DM.

There are several limitations in this study. First, we were unable to evaluate the causal relationship between leptin and lower-extremity artery calcification due to the cross-sectional design. Second, CT imaging, which is thought to reflect mainly plaque calcification, cannot precisely distinguish medial from intima calcification.

Our findings suggest that leptin could play a role in the development of lower-extremity artery calcification in T2DM through accelerating calcification of VSMCs via PI3K/Akt signaling pathway.

\section{REFERENCES}

1. Ogurtsova K, da Rocha Fernandes JD, Huang Y, Linnenkamp U, Guariguata L, Cho NH, et al. Idf Diabetes Atlas: Global Estimates for the Prevalence of Diabetes for 2015 and 2040. Diabetes Res Clin Pract (2017) 128:40-50. doi: 10.1016/j.diabres.2017.03.024

2. Agarwal S, Cox AJ, Herrington DM, Jorgensen NW, Xu J, Freedman BI, et al. Coronary Calcium Score Predicts Cardiovascular Mortality in Diabetes: Diabetes Heart Study. Diabetes Care (2013) 36:972-7. doi: 10.1016/j.diabres.2017.03.024

3. Stabley JN, Towler DA. Arterial Calcification in Diabetes Mellitus: Preclinical Models and Translational Implications. Arterioscler Thromb Vasc Biol (2017) 37:205-17. doi: 10.1161/ATVBAHA.116.306258

4. Pepe J, Cipriani C, Cilli M, Colangelo L, Minisola S. Adipokines and Bone Metabolism: An Interplay to Untangle. J Endocrinol Invest (2016) 39:1359-61. doi: 10.1007/s40618-016-0549-y

5. Kramer CK, von Mühlen D, Gross JL, Barrett-Connor E. A Prospective Study of Abdominal Obesity and Coronary Artery Calcium Progression in Older Adults. J Clin Endocrinol Met (2009) 94:5039-44. doi: 10.1210/jc.2009-1497

6. Ho JS, Cannaday JJ, Barlow CE, Willis B, Haskell WL, FitzGerald SJ. Comparative Relation of General, Central, and Visceral Adiposity Measures for Coronary Artery Calcium in Subjects Without Previous Coronary Events. Am J Cardiol (2009) 104:943-6. doi: 10.1016/j.amjcard.2009.05.047

7. Terry JG, Shay CM, Schreiner PJ, Jacobs DRJr., Sanchez OA, Reis JP, et al. Intermuscular Adipose Tissue and Subclinical Coronary Artery Calcification in Midlife: The Cardia Study (Coronary Artery Risk Development in Young Adults). Arterioscler Thromb Vasc Biol (2017) 37:2370-8. doi: 10.1161/ATVBAHA. 117.309633

\section{DATA AVAILABILITY STATEMENT}

The raw data supporting the conclusions of this article will be made available by the authors, without undue reservation.

\section{ETHICS STATEMENT}

The studies involving human participants were reviewed and approved by The Ethics Committee of Peking University International Hospital. The patients/participants provided their written informed consent to participate in this study.

\section{AUTHOR CONTRIBUTIONS}

Conception and design: SC and YQ. Analysis and interpretation of data: SC and XM. Investigation: SC, YC, SX, YL, NY, JS, and XM. Writing: SC. All authors contributed to the article and approved the submitted version.

\section{FUNDING}

This work was supported by the National Natural Science Foundation of China (81700779, 31872790), and by Peking University International Hospital Research Grant (YN2016QN05, YN2017QX02). The Outstanding Clinical Discipline Project of Shanghai Pudong and the program of Shanghai Municipal Health Commission (ZK2019B25).

8. Kuipers AL, Zmuda JM, Carr JJ, Terry JG, Nair S, Cvejkus R, et al. Association of Ectopic Fat With Abdominal Aorto-Illiac and Coronary Artery Calcification in African Ancestry Men. Atherosclerosis (2017) 263:198-204. doi: 10.1016/j.atherosclerosis.2017.06.030

9. Franssens BT, Nathoe HM, Visseren FL, van der Graaf Y, Leiner TSMART Study Group. Relation of Epicardial Adipose Tissue Radiodensity to Coronary Artery Calcium on Cardiac Computed Tomography in Patients At High Risk for Cardiovascular Disease. Am J Cardiol (2017) 119:1359-65. doi: 10.1016/ j.amjcard.2017.01.031

10. Zhang Y, Proenca R, Maffei M, Barone M, Leopold L, Friedman JM. Positional Cloning of the Mouse Obese Gene and its Human Homologue. Nature (1994) 372:425-32. doi: 10.1038/372425a0

11. Zhang Y, Chua S. Leptin Function and Regulation. Compr Physiol (2017) 8:351-69. doi: 10.1002/cphy.c160041

12. Andrade-Oliveira V, Câmara NO, Moraes-Vieira PM. Adipokines as Drug Targets in Diabetes and Underlying Disturbances. J Diabetes Res (2015) 2015:681612. doi: 10.1155/2015/681612

13. Vavruch C, Länne T, Fredrikson M, Lindström T, Östgren CJ, Nystrom FH. Serum Leptin Levels are Independently Related to the Incidence of Ischemic Heart Disease in a Prospective Study of Patients With Type 2 Diabetes. Cardiovasc Diabetol (2015) 14:62. doi: 10.1186/s12933-015-0208-1

14. Rodríguez AJ, Nunes Vdos S, Mastronardi CA, Neeman T, Paz-Filho GJ. Association Between Circulating Adipocytokine Concentrations and Microvascular Complications in Patients With Type 2 Diabetes Mellitus: A Systematic Review and Meta-Analysis of Controlled Cross-Sectional Studies. J Diabetes Complications (2016) 30:357-67. doi: 10.1016/j.jdiacomp. 2015.11.004 
15. Moonishaa TM, Nanda SK, Shamraj M, Sivaa R, Sivakumar P, Ravichandran K. Evaluation of Leptin as a Marker of Insulin Resistance in Type 2 Diabetes Mellitus. Int J Appl Basic Med Res (2017) 7:176-80. doi: 10.4103/ ijabmr.IJABMR_278_16

16. Yahagi K, Kolodgie FD, Lutter C, Mori H, Romero ME, Finn AV, et al. Pathology of Human Coronary and Carotid Artery Atherosclerosis and Vascular Calcification in Diabetes Mellitus. Arterioscler Thromb Vasc Biol (2017) 37:191-204. doi: 10.1161/ATVBAHA.116.306256

17. Parhami F, Tintut Y, Ballard A, Fogelman AM, Demer LL. Leptin Enhances the Calcification of Vascular Cells: Artery Wall as a Target of Leptin. Circ Res (2001) 88:954-60. doi: 10.1161/hh0901.090975

18. Qasim A, Mehta NN, Tadesse MG, Wolfe ML, Rhodes T, Girman C, et al. Adipokines, Insulin Resistance, and Coronary Artery Calcification. J Am Coll Cardiol (2008) 52:231-6. doi: 10.1016/j.jacc.2008.04.016

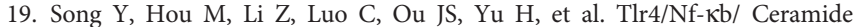
Signaling Contributes to Ox-LDL-induced Calcification of Human Vascular Smooth Muscle Cells. Eur J Pharmacol (2017) 794:45-51. doi: 10.1016/ j.ejphar.2016.11.029

20. Leopold JA. Vascular Calcification: Mechanisms of Vascular Smooth Muscle Cell Calcification. Trends Cardiovasc Med (2015) 25:264-74. doi: 10.1016/ j.tcm.2014.10.021

21. Chen G, Deng C, Li YP. Tgf- $\beta$ and BMP Signaling in Osteoblast Differentiation and Bone Formation. Int J Biol Sci (2012) 8:272-88. doi: 10.7150/ijbs.2929

22. Kusano T, Nakatani M, Ishiguro N, Ohno K, Yamamoto N, Morita M, et al. Desloratadine Inhibits Heterotopic Ossification by Suppression of BMP2Smad1/5/8 Signaling. J Orthop Res (2020) 1-8. doi: 10.1002/jor.24625

23. Yayama T, Uchida K, Kobayashi S, Kokubo Y, Sato R, Nakajima H, et al. Thoracic Ossification of the Human Ligamentum Flavum: Histopathological and Immunohistochemical Findings Around the Ossified Lesion. J Neurosurg Spine (2007) 7:184-193. doi: 10.3171/SPI-07/08/184

24. Kim HN, Min WK, Jeong JH, Kim SG, Kim JR, Kim SY, et al. Combination of Runx2 and BMP2 Increases Conversion of Human Ligamentum Flavum Cells Into Osteoblastic Cells. BMB Rep (2011) 44:446-51. doi: 10.5483/ BMBRep.2011.44.7.446

25. Gabbiani G. The Myofibroblast in Wound Healing and Fibrocontractive Diseases. J Pathol (2003) 200:500-3. doi: 10.1002/path.1427

26. Ye GJ, Nesmith AP, Parker KK. The Role of Mechanotransduction on Vascular Smooth Muscle Myocytes Cytoskeleton and Contractile Function. Anat Rec (2014) 297:1758-69. doi: 10.1002/ar.22983

27. Agatston AS, Janowitz WR, Hildner FJ, Zusmer NR, Viamonte M, Detrano R. Quantification of Coronary Artery Calcium Using Ultrafast Computed Tomography. J Am Coll Cardiol (1990) 15:827-32. doi: 10.1016/0735-1097 (90)90282-t

28. Cai Y, Xu MJ, Teng X, Zhou YB, Chen L, Zhu Y, et al. Intermedin Inhibits Vascular Calcification by Increasing the Level of Matrix GammaCarboxyglutamic Acid Protein. Cardiovasc Res (2010) 85:864-73. doi: $10.1093 / \mathrm{cvr} / \mathrm{cvp} 366$

29. Reaven GM, Knowles JW, Leonard D, Barlow CE, Willis BL, Haskell WL, et al. Relationship Between Simple Markers of Insulin Resistance and Coronary Artery Calcification. J Clin Lipidol (2017) 11:1007-12. doi: 10.1016/j.jacl.2017.05.013

30. Carmo LS, Burdmann EA, Fessel MR, Almeida YE, Pescatore LA, Farias-Silva E, et al. Expansive Vascular Remodeling and Increased Vascular Calcification Response to Cholecalciferol in a Murine Model of Obesity and Insulin Resistance. Arterioscler Thromb Vasc Biol (2019) 39:200-11. doi: 10.1161/ ATVBAHA.118.311880

31. Bessueille L, Magne D. Inflammation: A Culprit for Vascular Calcification in Atherosclerosis and Diabetes. Cell Mol Life Sci (2015) 72:2475-89. doi: 10.1007/s00018-015-1876-4

32. Byon $\mathrm{CH}$, Kim SW. Regulatory Effects of O-GlcNAcylation in Vascular Smooth Muscle Cells on Diabetic Vasculopathy. J Lipid Atheroscler (2020) 9:243-54. doi: 10.12997/jla.2020.9.2.243

33. Katz R, Budoff MJ, O’Brien KD, Wong ND, Nasir K. The Metabolic Syndrome and Diabetes Mellitus as Predictors of Thoracic Aortic Calcification as Detected by non-Contrast Computed Tomography in the Multi-Ethnic Study of Atherosclerosis. Diabetes Med (2015) 33:912-9. doi: 10.1111/ dme.12958
34. Chen NX, O’Neill K, Ak NK, Moe SM. Adipocyte Induced Arterial Calcification is Prevented With Sodium Thiosulfate. Biochem Biophys Res Commun (2014) 449:151-6. doi: 10.1016/j.bbrc.2014.05.005

35. Szulc P, Amri EZ, Varennes A, Panaia-Ferrari P, Fontas E, Goudable J, et al. Positive Association of High Leptin Level and Abdominal Aortic Calcification in Men- the Prospective Minos Study. Circ J (2018) 82:2954-61. doi: 10.1253/ circj.CJ-18-0517

36. Larsen BA, Laughlin GA, Cummins K, Barrett-Connor E, Wassel CL. Adipokines and Severity and Progression of Coronary Artery Calcium: Findings From the Rancho Bernardo Study. Atherosclerosis (2017) 265:1-6. doi: 10.1016/j.atherosclerosis.2017.07.022

37. Tanna N, Patel K, Moore AE, Dulnoan D, Edwards S, Hampson G. The Relationship Between Circulating Adiponectin, Leptin and Vaspin With Bone Mineral Density (Bmd), Arterial Calcification and Stiffness: A Cross-Sectional Study in Post-Menopausal Women. J Endocrinol Invest (2017) 40:1345-53. doi: 10.1007/s40618-017-0711-1

38. Wu J, Xu J, Wang K, Zhu Q, Cai J, Ren J, et al. Associations Between Circulating Adipokines and Bone Mineral Density in Patients With Knee Osteoarthritis: A Cross-Sectional Study. BMC Musculoskelet Disord (2018) 19:16. doi: 10.1186/s12891-018-1936-7

39. Biver E, Salliot C, Combescure C, Gossec L, Hardouin P, Legroux-Gerot I, et al. Influence of Adipokines and Ghrelin on Bone Mineral Density and Fracture Risk: A Systematic Review and Meta-Analysis. J Clin Endocrinol Metab (2011) 96:2703-13. doi: 10.1210/jc.2011-0047

40. Karwowski W, Naumnik B, Szczepański M, Myśliwiec M. The Mechanism of Vascular Calcification-a Systematic Review. Med Sci Monit (2012) 18:RA1RA11. doi: 10.12659/msm.882181

41. Demer LL. Effect of Calcification on In Vivo Mechanical Response of Rabbit Arteries to Balloon Dilation. Circulation (1991) 83:2083-93. doi: 10.1161/ 01.cir.83.6.2083

42. Trillhaase A, Haferkamp U, Rangnau A, Märtens M, Schmidt B, Trilck M et al. Differentiation of Human Ipscs Into Vsmcs and Generation of VSMCderived Calcifying Vascular Cells. Stem Cell Res (2018) 31:62-70. doi: 10.1210/ jc.2011-0047

43. Grootaert MOJ, Moulis M, Roth L, Martinet W, Vindis C, Bennett MR, et al. Vascular Smooth Muscle Cell Death, Autophagy and Senescence in Atherosclerosis. Cardiovasc Res (2018) 114:622-34. doi: 10.1161/ATVBAHA. 111.230904

44. Moe SM, O'Neill KD, Duan D, Ahmed S, Chen NX, Leapman SB, et al. Medial Artery Calcification in ESRD Patients is Associated With Deposition of Bone Matrix Proteins. Kidney Int (2002) 61:638-47. doi: 10.1046/j.1523-1755. 2002.00170.x

45. Chen S, Zhu H, Wang G, Xie Z, Wang J, Chen J. Combined Use of Leptin and Mechanical Stress has Osteogenic Effects on Ossification of the Posterior Longitudinal Ligament. Eur Spine J (2018) 27:1757-66. doi: 10.1007/s00586018-5663-4

46. Lin ME, Chen TM, Wallingford MC, Nguyen NB, Yamada S, Sawangmake C, et al. Runx2 Deletion in Smooth Muscle Cells Inhibits Vascular Osteochondrogenesis and Calcification But Not Atherosclerotic Lesion Formation. Cardiovasc Res (2016) 112:606-16. doi: 10.1093/cvr/cvw205

47. Wang S, Hu S, Wang J, Liu Y, Zhao R, Tong M, et al. Conditioned Medium From Bone Marrow-Derived Mesenchymal Stem Cells Inhibits Vascular Calcification Through Blockade of the BMP2-Smad1/5/8 Signaling Pathway. Stem Cell Res Ther (2018) 9:160. doi: 10.1186/s13287-018-0894-1

48. Kong Y, Liang Q, Chen Y, Yang P, Liu X, Li Y, et al. Hyaluronan Negatively Regulates Vascular Calcification Involving Bmp2 Signaling. Lab Invest (2018) 98:1320-32. doi: 10.1038/s41374-018-0076-x

49. Prawettongsopon C, Asawakarn S, Suthiphongchai T. Suppression of Prometastatic Phenotype of Highly Metastatic Androgen-Independent Rat Prostate Cancer MLL Cell Line by PI3K Inhibitor Ly294002. Oncol Res (2009) 17:301-9. doi: 10.3727/096504009787721195

50. He K, Yu X, Wang X, Tang L, Cao Y, Xia J, et al. Baicalein and Ly294002 Induces Liver Cancer Cells Apoptosis Via Regulating Phosphatidyl Inositol 3Kinase/Akt Signaling Pathway. J Cancer Res Ther (2018) 14:S519-25. doi: 10.4103/0973-1482.235356

51. Wang HF, Kuang MJ, Han SJ, Wang AB, Qiu J, Wang F, et al. Bmp2 Modified by the M6a Demethylation Enzyme ALKBH5 in the Ossification of the 
Ligamentum Flavum Through the AKT Signaling Pathway. Calcif Tissue Int (2020) 106:486-93. doi: 10.1007/s00223-019-00654-6

Conflict of Interest: The authors declare that the research was conducted in the absence of any commercial or financial relationships that could be construed as a potential conflict of interest.
Copyright (c) 2021 Chai, Chen, Xin, Yuan, Liu, Sun, Meng and Qi. This is an openaccess article distributed under the terms of the Creative Commons Attribution License (CCBY). The use, distribution or reproduction in other forums is permitted, provided the original author(s) and the copyright owner(s) are credited and that the original publication in this journal is cited, in accordance with accepted academic practice. No use, distribution or reproduction is permitted which does not comply with these terms. 